\title{
Surface-binding-energy shifts for sodium, magnesium, and aluminum metals
}

\author{
R. Kammerer, J. Barth, F. Gerken, and C. Kunz \\ II. Institut für Experimentalphysik, Universität Hamburg, D-2000 Hamburg 50, West Germany
}

S. A. Flodstrøm and L. I. Johansson

Department of Physics and Measurement Technology, Linköping University, S-58183 Linköping, Sweden

(Received 4 May 1982)

\begin{abstract}
The core-level binding-energy shifts for surface atoms relative to bulk atoms ("surface shifts") have been measured for the $2 p$ levels of $\mathrm{Na}(220 \mathrm{meV})$ and $\mathrm{Mg}$ (140 meV) using synchrotron-radiation-excited photoelectron spectroscopy. For Al, both a surface shift and a broadening are considered. The sign and magnitude of these shifts are compared with calculated values based on surface energies. The mean free path of the photoelectrons is determined by evaluation of the intensity ratio $I_{\text {bulk }}(2 p) / I_{\text {surface }}(2 p)$.
\end{abstract}

Recent photoelectron spectroscopy measurements have demonstrated that the core-level binding energies of surface atoms differ from those of bulk atoms. ${ }^{1-3}$ These surface shifts have been used to distinguish between different reconstruction models of metal and semiconductor surfaces and to study surface segregation in alloys. ${ }^{4}$ Only for the $5 d$ transition metals and the lanthanide series, however, enough experimental data have been reported to systematically compare calculated and measured surface shifts.

In a thermodynamic model, the surface shift $\Delta E_{c}$ can be expressed as ${ }^{5,6}$

$$
\Delta E_{c} \simeq E_{s}(Z+1)-E_{s}(Z),
$$

with $E_{s}(Z)$ the surface energy of the element with mass number $Z$. Using this model and surface energies, either estimated from bulk cohesive energies ${ }^{5}$ or from tight-binding calculations of the surfaces, ${ }^{6,7}$ the surface shifts have been obtained for the $5 d$ transition metals. A comparison between experimental and calculated shifts has been encouraging; the trend (a change of sign in the middle of the $5 d$ series) has been found in experiments, the magnitude compares reasonably well and the predicted difference in surface shifts of the different crystal faces of the same metal has been found. ${ }^{6}$ The thermodynamic model appears to be a promising theory and gives reason to expect surface shifts on free-electron-like metals as well. Therefore, it is challenging to search for them.

The present Communication reports measurements on the $2 p$ levels of $\mathrm{Na}, \mathrm{Mg}$, and $\mathrm{Al}$. We studied evaporated films and single crystals $[\mathrm{Mg}(0001)$, $\mathrm{Al}(100)$, and Al(111)]. Two aspects of the surface shifts are treated: First, we correlate sign and value of the observed surface shift with calculated values using relation (1) and surface energies obtained from theoretical calculations ${ }^{8,9}$ or from other types of experiments. $^{10-12}$ Second, we estimate the mean free path of the photoelectrons from the bulk-to-surface intensity ratio of the $2 p$ peaks $\left(I_{B} / I_{S}\right)$.

The experiments were performed at the Hamburger Synchrotronstrahlungslabor (HASYLAB) using synchrotron radiation of the storage ring DORIS. Monochromatic radiation from the FLIPPER monochromator was used in the energy range of 36-200 $\mathrm{eV}$ and the photoelectrons were analyzed in a commerical cylindrical mirror analyzer. The overall instrumental resolution was $0.09-0.19 \mathrm{eV}$ in all data presented. The films were evaporated onto stainlesssteel $(\mathrm{Mg}, \mathrm{Al})$ or oxygen-free high-conductivity copper $(\mathrm{Na})$ substrates under UHV conditions and spectra recorded at 300 and $100 \mathrm{~K}$. The single crystals were polished in a standard way, "sputter" cleaned, and annealed. All data on single crystals were recorded at $300 \mathrm{~K}$.

Figure 1 shows spectra of the $\mathrm{Na} 2 p$ level at several excitation energies. To fit the experimental spectra, a procedure shown in the $100-\mathrm{eV}$ spectrum of Fig. 1 was used: We assumed two sets of peaks, $2 p_{3 / 2,1 / 2}$ (bulk) and $2 p_{3 / 2,1 / 2}$ (surface) with the spinorbit intensity ratio fixed to $2: 1$. According to the theory of free-electron-like metals, we used Doniach-Sunjic line shapes convoluted with a Gaussian of temperature-dependent width to consider phonon broadening. ${ }^{13}$ The sum of the doublets was convoluted with a Gaussian to take the instrumental resolution into account. Thus the intensity ratio $I_{B} / I_{S}$ and the surface shift $E_{c}$ (bulk) $-E_{c}$ (surface) were used as main parameters. Experimental spectra could not satisfactorily be fitted using an identical set of parameters for bulk and surface doublets. We attribute this to the structure dependence of the surface shift. ${ }^{14}$ The random orientation of crystallites at the surface of the evaporated film leads to a multitude of surface doublets with different surface shifts. Including a second or even third surface doublet into the fit introduces so many new free parameters (for want of single-crystal data), that results become meaningless. 


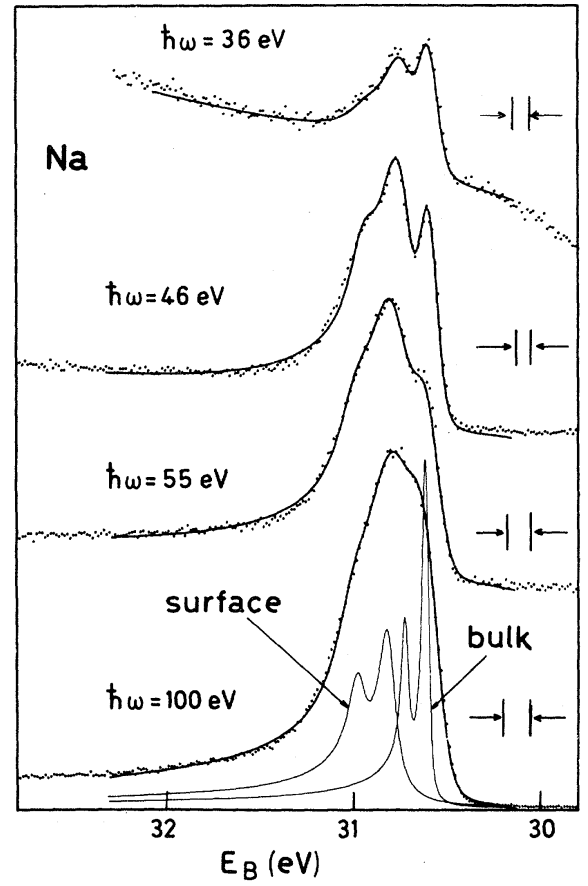

FIG. 1. Spectra of the Na $2 p$ level taken at $100 \mathrm{~K}$ and several photon energies to illustrate the energy-dependent prominence of a surface doublet. The fit procedure, as explained in the text, is shown at the $100-\mathrm{eV}$ spectrum. For clarity, the convolution regarding instrumental resolution has not yet been carried out.

We have, therefore, chosen a different approach which we regard as more suitable to determine surface shifts on polycrystalline films: Parameters of the bulk doublet were taken from x-ray photoemission spectroscopy (XPS) measurements ${ }^{13}$ which are not surface sensitive. Width and asymmetry of just one surface doublet and the position of individual lines on the energy scale were adjusted to achieve to the best fit possible, and experimental results were extracted from the entirety of all spectra. In particular, we found a spin-orbit splitting of $140 \pm 23 \mathrm{meV}$ with the bulk value generally lower than the mean which is caused by the restraint to one surface doublet in our fitting procedure. The $\mathrm{Na}$ data are confirmed by evaluation of the $2 s$ peak. It can be fitted using the parameters determined from the $2 s$ spectra with a width corresponding to XPS data. Compatible results have been obtained using yield spectroscopy at the edge as in Refs. 1 and 15. Since our experimental setup permits more accurate evaluation of energy distribution curves, we will focus on these results. The experimental data are compiled in Table I.

In order to discuss the surface shifts using relation (1) we need the surface energies of $\mathrm{Na}, \mathrm{Mg}, \mathrm{Al}$, and Si. Surface energies have been calculated in the density functional formalism applied to the inhomogeneous electron gas. By including pseudopotentials of the metal ions and the classical electrostatic cleavage energy into the model and improving the numerical calculation of the exchange energy, good agreement with experiments for $\mathrm{Na}, \mathrm{Mg}$, and $\mathrm{Al}$ was achieved. ${ }^{8,9}$ The experimental surface energies for metals have usually been obtained at the melting point. ${ }^{10}$ Only few experiments report on data measured at low temperatures. ${ }^{11}$ In Table I surface energies derived in several different ways are shown together with expected shifts for $\mathrm{Na}, \mathrm{Mg}$, and $\mathrm{Al}$, which we calculated according to relation (1).

Spectra of $\mathrm{Na}$ (Fig. 1) and $\mathrm{Mg}$ (Fig. 2) clearly show the presence of an energy-shifted surface doublet. On the other hand, the surface sensitive (100 and $120 \mathrm{eV}$ ) spectra of $\mathrm{Al}(100)$ (Fig. 3) just show a broadening of ambiguous origin. This was interpreted by Eberhardt et al. ${ }^{15}$ to be a broadening due to crystal-field splitting. Very recently, Chiang and Eastman ${ }^{1}$ reported a surface shift of $-57 \mathrm{meV}$ also measured on an $\mathrm{Al}(100)$ face in yield spectroscopy. A theoretical study ${ }^{16}$ resulted in both a splitting and a

TABLE I. Core-level binding energies $E_{c}(\mathrm{eV})$, surface shifts $\Delta E_{c}(\mathrm{meV})$, width (meV), theoretical and experimental surface energies $E_{s}$ (meV/atom) for $\mathrm{Na}, \mathrm{Mg}$, and $\mathrm{Al}$. Uncertainty of our experimental results is $<10 \%$ and $\pm 0.05 \mathrm{eV}$ for $E_{c}$. The evaporated $\mathrm{Al}$ film yields the same results as the $(100)$ face. The $(100)$ spectra can be fitted either assuming a surface shift or a surface sensitive broadening. Relation (1) has been used to calculate surface shifts. The surface energy of Si has not been calculated. (FWHM is full width at half maximum.)

\begin{tabular}{lcccccccc}
\hline \hline & $\begin{array}{c}E_{c} \\
\text { (expt.) }\end{array}$ & $\begin{array}{c}\text { FWHM } \\
\text { Bulk }\end{array}$ & $\begin{array}{c}\text { FWHM } \\
\text { Surface }\end{array}$ & $\begin{array}{c}\Delta E_{c} \\
\text { (expt.) }\end{array}$ & $\begin{array}{c}\Delta E_{c} \\
\text { (calc.) }\end{array}$ & $\begin{array}{c}E_{s} \\
\text { (theory) }\end{array}$ & $\begin{array}{c}E_{s} \text { (expt.) } \\
\text { (Refs. 11 and 12) }\end{array}$ & $\begin{array}{c}E_{s} \text { (expt.) } \\
\text { (Ref. 12) }\end{array}$ \\
\hline $\mathrm{Na}$ & 30.60 & 24 & 90 & 220 & 199 & 205 & 215 & 184 \\
$\mathrm{Mg}$ & 49.60 & 68 & 100 & 140 & 105 & 404 & 401 & 383 \\
$\mathrm{Al}(100)$ splitting & 72.71 & 76 & 110 & $(-120)$ & $(-106)$ & 505 \\
$\mathrm{Al}(100)$ broadening & 72.76 & 176 & $\ldots$ & $\ldots$ & & 518 & 481 \\
$\mathrm{Al}(111)$ & 72.71 & 83 & $\ldots$ & $\ldots$ & $(3)$ & 372 \\
$\mathrm{Si}$ & & & & & & & 375 \\
\hline \hline
\end{tabular}




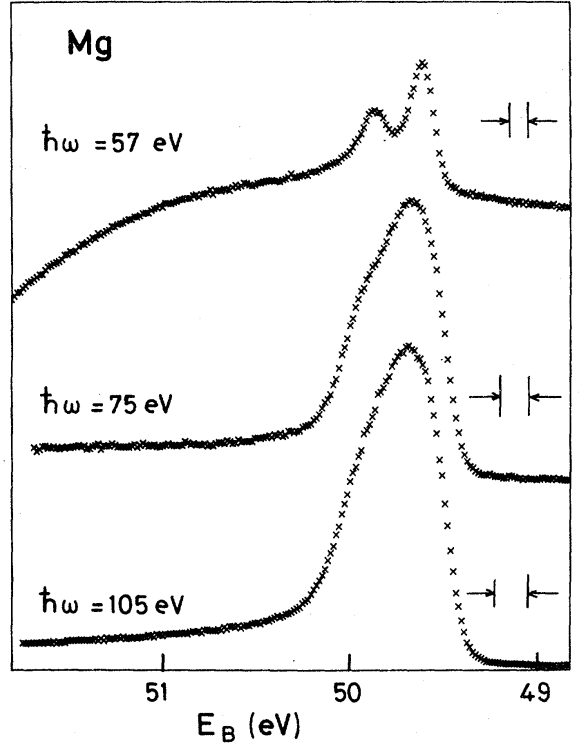

FIG. 2. Spectra of $\mathrm{Mg}$ at $100 \mathrm{~K}$ show the surface doublet, but not as clearly as in Na. This is due to the reduced surface shift and the larger spin-orbit splitting.

surface shift of $-120 \mathrm{meV}$ for the top layer atoms. We are able to fit the experimental curve by adding a surface doublet shifted by $-120 \mathrm{meV}$, but we can also reproduce the experimental result using just one doublet which is allowed to broaden as measurements become surface sensitive. Experimental uncertainty

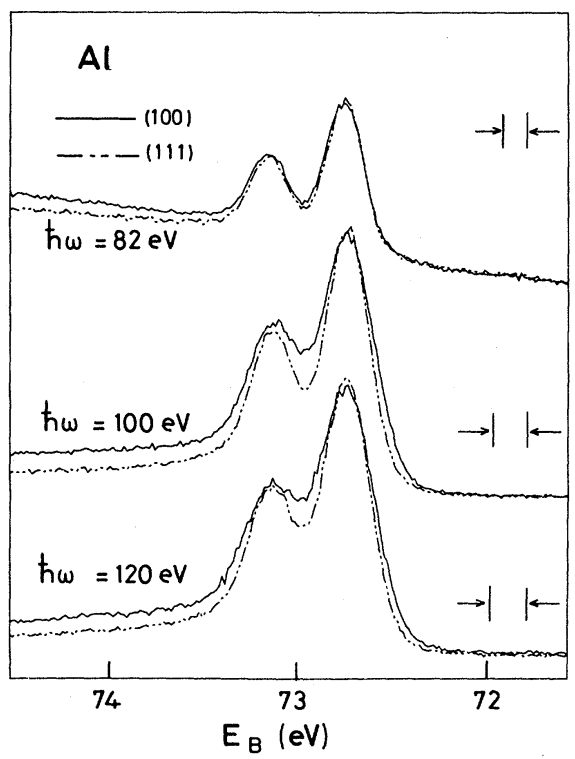

FIG. 3. Spectra of Al $2 p$ taken from different crystal faces. In the surface-sensitive spectra at 100 and $120 \mathrm{eV}$, the (100) spectrum (solid) is broadened as compared with the (111) spectrum (dashed-dotted). prevents reliable judgment from the expected shift of the curves' center of gravity. Therefore, our data on $\mathrm{Al}(100)$ do not enable us to resolve the discussion beyond doubt.

Measurements on the $\mathrm{Al}(111)$ face, however, show no surface sensitivity at all. All spectra can be fitted using just one doublet with constant full width at half maximum (Fig. 3). Since the theoretical value of the surface energy of $\mathrm{Al}(111)$ is about $130 \mathrm{meV} / \mathrm{atom}$ smaller than the (100) value, ${ }^{8}$ an extremely small or vanishing surface shift is expected from the theoretical model. If the reader follows the contention that a broadening mechanism existing only on the (100) [and maybe on the (110)] face is unlikely, the line shapes of the $2 p$ levels appear to be given by a superposition of a bulk and a surface doublet.

The intensity ratio $I_{B} / I_{S}$ has so far been used to estimate the number of surface atoms versus bulk atoms for reconstructed surfaces. On the other hand, by comparing the $I_{B} / I_{S}$ ratios for different kinetic energies of the photoelectrons we will be able to express the mean free path in units of atomic layers as a function of electron kinetic energy. ${ }^{2}$ This technique is demonstrated on $\mathrm{Na}$. It has the largest surface shift which makes the determination of $I_{B} / I_{S}$ more accurate. Figure 4 shows the mean free path versus kinetic energy over a wide energy range. The minimum is clearly discernible at 20 to $40 \mathrm{eV}$. For higher kinetic energies our results can be compared with the calculation by Penn ${ }^{17}$ who used a freeelectron gas model. Calculations of the mean free path for low kinetic energies are few and disagreeing. Present results may stimulate new work.

In summarizing our results, we have for the first time clearly observed the existence of surface shifts on free-electron-like metals, namely, $\mathrm{Na}$ and $\mathrm{Mg}$. The shifts can be successfully correlated with surface

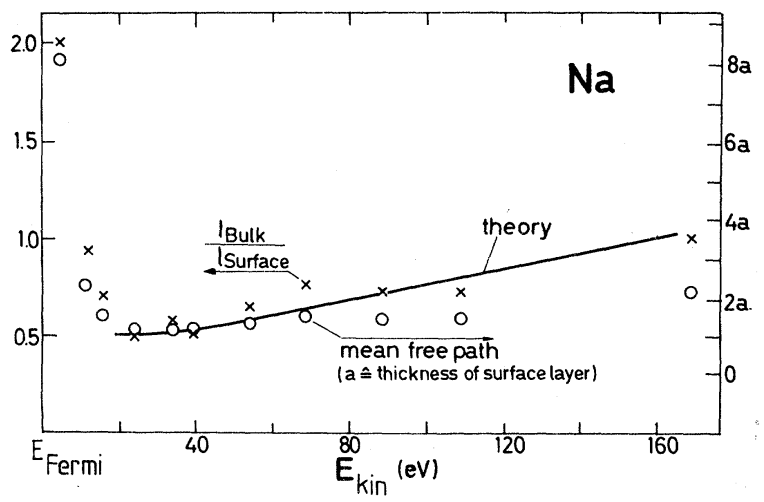

FIG. 4. Mean free path (0) of the photoelectrons is determined from the intensity ratio of the bulk and surface doublets $(x)$ and expressed in units of the distance between layers. To compare with theory, we assumed a bcc (110) packing resulting in an $a=3 \AA$. 
energy data by the Johansson-Marrtensson theory. ${ }^{5}$ The surface shift of $\mathrm{Al}(111)$ is practically zero, while the case of $\mathrm{Al}(100)$ appears to be more involved. There is some indication, however, that the theoretical concept also holds for Al.

\section{ACKNOWLEDGMENTS}

This work was in part supported by Swedish Natural Science Research Council (NFR). We thank B. Blume for encouragement during the measurements.
${ }^{1}$ T.-C. Chiang and D. E. Eastman, Phys. Rev. B $\underline{23}, 6836$ (1981), and references cited therein.

${ }^{2}$ R. Kammerer et al., Solid State Commun. 41, 435 (1982).

${ }^{3} \mathrm{~F}$. Gerken et al., Surf. Sci. (in press).

${ }^{4}$ L. Johansson et al., Solid State Commun. 41, 427 (1982).

${ }^{5}$ B. Johansson and N. Mårtensson, Phys. Rev. B 21, 4427 (1980).

${ }^{6}$ A. Rosengren and B. Johansson, Phys. Rev. B 22, 3706 (1980).

${ }^{7}$ M. C. Desjonqueres and F. Cyrot-Lackman, Surf. Sci. $\underline{50}$, 257 (1975).

${ }^{8}$ N. D. Lang and W. Kohn, Phys. Rev. B 1, 4555 (1970).

${ }^{9}$ J. H. Rose, H. B. Shore, D. Geldart, and M. Rasolt, Solid State Commun. 19, 619 (1976).
${ }^{10}$ J. Bodanski and H. E. J. Shins, J. Inorg. Nucl. Chem. 30, 2331 (1968)

${ }^{11} \mathrm{H}$. Wawra, Z. Metallkd. 66, 395 and 492 (1975).

${ }^{12}$ W. Tyson and N. Miller, Surf. Sci. 62, 267 (1977).

${ }^{13}$ P. H. Citrin, J. K. Wertheim, and Y. Baer, Phys. Rev. B 16,4256 (1979).

${ }^{14}$ P. Heimann, J. F. van der Veen, and D. E. Eastman, Solid State Commun. 38, 595 (1981).

${ }^{15}$ W. Eberhardt, G. Kalkoffen, and C. Kunz, Solid State Commun. 32, 901 (1979).

${ }^{16}$ E. Wimmer, M. Weinert, A. J. Freeman, and H. Krakauer, Phys. Rev. B 24, 2292 (1981).

${ }^{17}$ D. R. Penn, Phys. Rev. B 13, 5248 (1976). 